\title{
Qualidade física de um Latossolo Vermelho sob sistemas de integração lavoura-pecuária no Cerrado
}

\author{
Robélio Leandro Marchão(1), Luiz Carlos Balbino(2), Euzebio Medrado da Silva(1), \\ João de Deus Gomes dos Santos Junior ${ }^{(1)}$, Marcos Aurélio Carolino de Sá(1), Lourival Vilela ${ }^{(1)}$ e Thierry Becquer ${ }^{(3)}$ \\ (1)Embrapa Cerrados, BR 020, Km 18, Caixa Postal 08223, CEP 73310-970 Planaltina, DF. E-mail: robelio.leandro@cpac.embrapa.br, \\ euzebio@cpac.embrapa.br, jdsantos@cpac.embrapa.br, carolino@cpac.embrapa.br, Ivilela@cpac.embrapa.br (2)EmbrapaTransferência de \\ Tecnologia, Parque Estação Biológica, Ed. Embrapa Sede, CEP 70770-901 Brasília, DF. E-mail: luizcarlos.balbino@embrapa.br (3)Institut \\ de Recherche pour le Développement, Unité Mixte de Recherche 137, 32 Avenue Henri Varagnat, 93143 Bondy, France. \\ E-mail: becquer@cpac.embrapa.br
}

\begin{abstract}
Resumo - O objetivo deste trabalho foi avaliar o impacto de sistemas de integração lavoura-pecuária sobre atributos físico-hídricos do solo e avaliar o potencial uso desses atributos como indicadores da qualidade física de um Latossolo. As amostras de solo foram coletadas nas camadas 0-5, 10-15, 20-25 e 40-45 cm. Todos os sistemas de uso e manejo do solo alteraram significativamente a densidade, umidade volumétrica, resistência à penetração, porosidade total, macroporosidade, microporosidade efetiva e água prontamente disponível do solo. Foram observados incrementos na resistência à penetração e na densidade do solo em todos os sistemas em comparação ao cerrado nativo. A compactação resultante do pisoteio animal durante quatro anos da fase pastagem, nos sistemas de integração lavoura-pecuária, não atingiu valores críticos, que pudessem limitar cultivos anuais subseqüentes. A porosidade total e a macroporosidade foram maiores no cerrado e nos sistemas em plantio direto em relação aos em preparo convencional. Os atributos avaliados são indicadores confiáveis da qualidade físico-hídrica do solo.
\end{abstract}

Termos para indexação: sistemas de cultivo, resistência à penetração, porosidade, retenção de água, qualidade do solo.

\section{Soil physical quality under crop-livestock management systems in a Cerrado Oxisol}

\begin{abstract}
The objective of this work was to analyze the effect of integrated crop-livestock systems on soil hydraulic properties, and test the potential use of these properties as physical quality indicators of a Cerrado Oxisol. The field study was conducted at Embrapa Cerrados, Planaltina, DF, Brazil, considering soil layers of: 0-5, 10-15, 20-25 and 40-45 cm. Crop-livestock systems caused significant changes in soil bulk density, volumetric soil-water content, soil penetration resistance, total porosity, macroporosity, effective microporosity, unsaturated pores and available water. An increase was observed in both soil resistance and bulk density for all systems in comparison to the cerrado. However, higher soil resistance did not appear as a limiting factor for annual crops growth in a rotation sequence. Total porosity and macroporosity were higher in the cerrado, and in the systems with no-tillage in relation to conventional tillage. Soil penetration resistance, macroporosity, bulk density and available soil-water properties are reliable indicators of the soil physical quality.
\end{abstract}

Index terms: crop-pasture rotation, soil penetration resistance, porosity, water retention, soil quality.

\section{Introdução}

A qualidade física do solo (QFS) é fundamental para a sustentabilidade global dos agroecossistemas (Millennium Ecosystem Assessment, 2005). O conceito de QFS é dinâmico e abrangente e tem sido discutido desde o início da década de 90 . No entanto, a QFS é ainda uma área de pesquisa em expansão (Lal, 2000; Reynolds et al., 2002).

Dos 204 milhões de hectares da Região do Cerrado, Sano et al. (2000) estimaram que aproximadamente 50 milhões são ocupados por pastagens cultivadas. Atualmente, estima-se que entre $70 \mathrm{e} 80 \%$ das pastagens cultivadas encontra-se em algum estágio de degradação. Nessas pastagens, devido ao manejo inadequado, observa-se redução na porosidade total das camadas superficiais do solo que afetam a reserva de água disponível, especialmente à medida que a meso e a microporosidade do solo se reduzem (Balbino et al., 2001).

Atributos do solo, tais como a densidade, a porosidade, a condutividade hidráulica, a curva característica de retenção de água (Balbino et al., 2004) 
e a resistência à penetração (Imhoff et al., 2000), têm sido comumente utilizados como indicadores de qualidade física, pela relativa facilidade de determinação e pelo baixo custo de obtenção das medidas. Além da comparação entre os sistemas de manejo e de uso do solo (Oliveira et al., 2004), os atributos físicos também têm sido utilizados para estudar o efeito da conversão de áreas nativas em lavouras ou pastagens (Leão et al., 2006).

Em diferentes regiões do mundo, existe um consenso de que períodos de utilização com pastagens perenes, gramíneas e ou leguminosas, condicionam melhorias na qualidade do solo e na produtividade de lavouras subseqüentes (García-Préchac et al., 2004). A estabilidade de agregados, a macroporosidade e a condutividade hidráulica podem aumentar rapidamente com a inclusão de pastagens na rotação com culturas devido à combinação de três efeitos principais: ausência de preparo durante o ciclo da pastagem, presença de um denso sistema radicular, que atua como agente agregante, e maior atividade da macrofauna do solo em pastagens (Marchão, 2007). Este processo é rapidamente revertido quando o solo volta a ser preparado.

Estudos sobre a utilização de sistemas de integração lavoura-pecuária (ILP) e seu impacto sobre os atributos físico-hídricos do solo são ainda incipientes. A identificação de indicadores sensíveis a esse impacto é fundamental na comparação das diversas práticas de manejo sobre o comportamento físicohídrico do solo. Assim, objetivou-se analisar e comparar o efeito de sistemas de manejo e uso do solo, incluindo sistemas de integração lavourapecuária, sobre suas propriedades físico-hídricas e avaliar o potencial uso dessas propriedades como indicadoras da qualidade física de um Latossolo Vermelho do Cerrado.

\section{Material e Métodos}

O estudo foi realizado em um experimento de integração lavoura-pecuária (ILP) da Embrapa Cerrados em Planaltina, DF, em um Latossolo Vermelho (Embrapa, 2006) fase cerrado típico, cuja fração argila é predominantemente composta por gibbsita, caulinita, e óxidos de ferro (Chapuis-Lardy et al., 2002). Possui, em média, na camada de $0-45 \mathrm{~cm}, 62,22 \%$ de argila, 7,41\% de silte, 30,35\% de areia e 2,69\% de matéria orgânica.

Em 1991, a vegetação nativa foi removida e o solo preparado com arado de disco seguido de grade aradora e corrigido com calcário dolomítico na dose de $3,4 \mathrm{Mg} \mathrm{ha}^{-1}$ antes da instalação do experimento. Em 1999, foi realizada nova calagem na dose de $1,0 \mathrm{Mg} \mathrm{ha}^{-1}$, na superfície nas áreas em plantio direto, e incorporado nas áreas em plantio convencional. A área de cada parcela experimental foi de $50 \mathrm{~m}$ de comprimento por $40 \mathrm{~m}$ de largura.

Foram estudados oito tratamentos, compostos pela combinação de tipos de uso (cultivos contínuos e integração lavoura-pecuária) e sistemas de preparo do solo na fase lavoura (Tabela 1). Nos sistemas de integração lavoura-pecuária, a alternância entre pastagem e lavoura, e vice-versa, ocorreu a cada quatro anos, com o objetivo de recuperar a fertilidade das pastagens por meio da fertilização das culturas anuais. No sistema LP-D, devido à ausência de preparo, a forrageira foi implantada em semeadura direta no último ano de cultivo do ciclo lavoura. As sementes da forrageira foram misturadas ao fertilizante utilizado na adubação da cultura anual. No sistema LP-C, foi realizada gradagem após a colheita da cultura no último ano do ciclo LP, para posterior semeadura da forrageira. Uma área de Cerrado nativo foi mantida como referência para comparação com as áreas cultivadas. Nas áreas sob pastagem, foi utilizado o sistema de lotação rotacionado, com períodos de ocupação e descanso de

Tabela 1. Sistemas de manejo e uso do solo estudados no experimento de integração lavoura-pecuária.

\begin{tabular}{|c|c|c|c|}
\hline Sistema de manejo & $\begin{array}{l}\text { Tipo de } \\
\text { preparo }^{(1)}\end{array}$ & Cultivo e idade na amostragem ${ }^{(2)}$ & Representação \\
\hline Cerrado & Ausente & Vegetação nativa & CER \\
\hline Pastagem contínua & Ausente & Brachiaria decumbens $13^{\circ}$ ano & PAST \\
\hline \multirow[t]{2}{*}{ Rotação pastagem-lavoura } & $\mathrm{PD}$ & Panicum maximum $4^{\mathrm{o}}$ ano (soja 4 anos) & PL-D \\
\hline & $\mathrm{PC}$ & Panicum maximum $4^{\circ}$ ano (soja 4 anos) & PL-C \\
\hline \multirow[t]{2}{*}{ Rotação lavoura-pastagem } & PD & Brachiaria brizantha $1^{\circ}$ ano (soja 4 anos) & LP-D \\
\hline & $\mathrm{PC}$ & Solo preparado (soja 4 anos) & LP-C \\
\hline \multirow[t]{2}{*}{ Lavoura contínua } & PD & Lavoura de soja $4^{\circ}$ ano & L-D \\
\hline & $\mathrm{PC}$ & Lavoura de soja $4^{\circ}$ ano & $\mathrm{L}-\mathrm{C}$ \\
\hline
\end{tabular}

${ }^{(1)} \mathrm{PD}$, plantio direto; PC, plantio convencional. ${ }^{(2)} \mathrm{O}$ texto entre parênteses indica a cultura antecessora no sistema de rotação. 
14 dias. A oferta de forragem -8 a $10 \mathrm{~kg}$ de matéria verde por $100 \mathrm{~kg}$ de peso vivo - foi constante e ajustada a cada 28 dias.

Foram coletadas, em cada tratamento, seis amostras indeformadas de solo, em novembro de 2004, com o auxílio de anéis volumétricos de $5,0 \mathrm{~cm}$ de altura por $5,0 \mathrm{~cm}$ de diâmetro, nas camadas de $0-5,10-15,20-25$ e 40-45 cm, perfazendo um total de 192 amostras. Os cilindros foram coletados em um ponto central da parcela, em trincheiras de 2,0x1,5x1,0 m. O delineamento experimental utilizado foi o inteiramente casualizado. Por ocasião da amostragem, medidas de resistência à penetração $(\mathrm{RP})$ e umidade volumétrica $\left(\theta_{\mathrm{v}}\right)$ foram tomadas aleatoriamente, nas mesmas profundidades. A RP foi medida utilizando um penetrógrafo dinamométrico ligado a um registrador analógico. No momento da amostragem, o solo encontrava-se com umidade próxima da capacidade de campo. As medidas foram realizadas em três pontos ao redor das trincheiras e em cada ponto foram feitas quatro leituras totalizando 12 repetições.

De cada profundidade, três cilindros foram aleatoriamente selecionados e utilizados para determinação da curva de retenção de água do solo. As amostras foram previamente saturadas e submetidas ao processo de centrifugação (Silva \& Azevedo, 2002) para determinação do conteúdo da água do solo em equilíbrio com as tensões correspondentes a 1, 3, 6, 10, $33,84,406,1.027,1.515 \mathrm{kPa}$ (Silva et al., 2006). Ao final deste processo, as amostras foram secadas em estufa a $105^{\circ} \mathrm{C}$ para determinação da massa seca, da densidade do solo (Ds) e dos conteúdos gravimétricos de água $(\theta)$ associados à cada tensão aplicada $(\mathrm{kPa})$. Para cada valor de $\theta$, foi calculado o conteúdo relativo de água $\left(\theta_{\text {rel }}\right)$, utilizando-se a fórmula $\theta_{\text {rel }}=\left(\theta-\theta_{1.515 \mathrm{kPa}}\right) /\left(\theta_{\mathrm{s}}-\theta_{1.515 \mathrm{kPa}}\right)$, em que $\theta_{\mathrm{s}}$ é o conteúdo gravimétrico de água na saturação, determinado por meio da pesagem direta da amostra do solo saturado; $\theta_{1.515 \mathrm{kPa}}$ é o conteúdo gravimétrico da água do solo, correspondente à tensão de $1.515 \mathrm{kPa}$; e a diferença $\theta_{\mathrm{S}}-\theta_{1.515 \mathrm{kPa}}$ é a saturação efetiva $\left(\mathrm{S}_{\mathrm{e}}\right)$ de água no solo. Os valores de $\theta_{\text {rel }}$ foram ajustados a uma função contínua, utilizando o seguinte modelo não-linear (Assouline et al., 1998) de curva de retenção da água do solo (CRA):

$\mathrm{CRA}=1-\left[\exp \left(-\alpha\left(1 / \mathrm{T}-1 / \mathrm{T}_{1.515 \mathrm{kPa}}\right)^{\mathrm{n}}\right)\right]$, em que $\mathrm{n}$ e $\alpha$ são os parâmetros de ajuste do modelo e T é a tensão $(\mathrm{kPa})$ em um ponto qualquer da curva menor ou igual a $1.515 \mathrm{kPa}$. Os parâmetros n e $\alpha$ do modelo de Assouline et al. (1998) foram obtidos por meio da rotina Solver da planilha eletrônica Excel.
Em cada um dos oito sistemas de manejo e quatro profundidades, foi construída uma CRA, o que resultou em 32 curvas. Essas curvas foram comparadas entre si, por profundidade, utilizando o procedimento descrito por Silva \& Azevedo (2002), tendo por base a soma de quadrados dos erros (SQerro) obtidos no ajuste da CRA. A partir dessa comparação, foi possível determinar um valor de $\mathrm{F}$, com 2 graus de liberdade no numerador e 12 no denominador, a $1 \%$ de probabilidade de erro, correspondente a cada uma das 28 comparações entre os sistemas de manejo e por profundidade.

$\mathrm{Na}$ separação entre macro e microporos, foi utilizado o conteúdo de água correspondente à tensão de $6 \mathrm{kPa}$ (Embrapa, 1997). Além da porosidade total (PT), macroporosidade (MAC) e microporosidade (MIC), foi também quantificada a microporosidade efetiva (MIE), definida pela diferença entre os conteúdos de água nas tensões de $6 \mathrm{kPa}$ e $1.515 \mathrm{kPa}$. $\mathrm{O}$ volume de poros insaturados (PI) foi calculado como a diferença entre a porosidade total (teórica) e o conteúdo de água na saturação medida. A água prontamente disponível (APD) foi calculada pela diferença entre o conteúdo volumétrico de água nas tensões de 6 e $1.000 \mathrm{kPa}$ (Silva \& Resck, 1981). Os valores de Ds, $\theta_{\mathrm{v}}$, RP, PT, MAC, MIC, MIE, PI, APD foram submetidos à análise de variância a fim de verificar o efeito dos sistemas de manejo e da profundidade. Coeficientes de correlação de Pearson foram estimados como medida de dependência entre as variáveis RP, Ds e $\theta_{v}$. Os tratamentos foram comparados pelo teste de Tukey, a $5 \%$ de probabilidade.

Finalmente, a qualidade físico-hídrica do solo foi avaliada com o uso de modelos gráficos, do tipo radial, em que cada parâmetro foi locado em um dos seus raios (Costa et al., 2006). Os parâmetros físico-hídricos medidos na testemunha (CER) foram adotados como referência. $\mathrm{O}$ indicador numérico para expressar o efeito do tratamento sobre cada parâmetro utilizado (MAC, RP, Ds e MIE) foi definido como sendo a diferença entre a unidade e o quociente do valor do parâmetro considerado e seu respectivo valor de referência (CER), multiplicada por 100. Desse modo, o valor desse indicador numérico de qualidade era sempre zero no tratamento de referência; positivo em qualquer valor superior ao de referência e negativo em qualquer valor inferior. Por meio desse tipo de apresentação, foi possível construir um esquema gráfico radial para avaliar qualitativamente a tendência do impacto em 
relação ao uso contínuo dessas áreas na qualidade físico-hídrica do solo.

\section{Resultados e Discussão}

Os valores de Ds (Tabela 2) foram semelhantes aos valores encontrados em Latossolos do Cerrado sob sistemas agropastoris (Tormena et al., 1999; Balbino et al., 2004). Na camada de $0-5 \mathrm{~cm}$, as maiores Ds foram observadas nos sistemas PL-D e PL-C. Os quatro anos da pastagem de Panicum maximum na rotação, em conjunto com o plantio direto, provocaram, portanto, uma compactação superficial em relação às demais pastagens e às áreas em plantio convencional.

Estes resultados corroboram os observados por Spera et al. (2004), que também relataram aumento de densidade no perfil do solo sob pastagens e em plantio direto. O P. maximum, por seu hábito de crescimento cespitoso, apresenta alto porcentual de solo descoberto entre touceiras, o que torna o solo mais susceptível à ação da compactação superficial pelo pisoteio animal (Imhoff et al., 2000). O mesmo resultado foi observado no sistema LP-D, em que a Brachiaria brizantha foi plantada associada à cultura da soja. Em relação à testemunha (CER), todos os sistemas de manejo, incluindo a pastagem contínua de Brachiaria decumbens, apresentaram incremento na densidade na camada de $0-5 \mathrm{~cm}$. Os sistemas em pastagem (PAST, PL-C, LP-D) apresentaram, no entanto, decréscimo na Ds em profundidade. Por sua vez, os sistemas em lavoura não apresentaram a mesma tendência. Diferentemente do que ocorreu nas pastagens, no sistema LP-C a Ds aumentou em profundidade, provavelmente resultado da compactação em

Tabela 2. Valores médios de atributos físico-hídricos de acordo com sistemas de manejo e uso do solo e da profundidade de $\operatorname{amostragem}^{(1)}$.

\begin{tabular}{|c|c|c|c|c|c|c|c|c|c|}
\hline Atributos $^{(2)}$ & $\begin{array}{l}\text { Camada } \\
\text { (cm) }\end{array}$ & CER & PAST & PL-C & PL-D & LP-C & LP-D & L-C & L-D \\
\hline $\mathrm{DS}$ & $0-5$ & $0,87 \mathrm{~d}$ & $1,13 \mathrm{abc}$ & $1,19 \mathrm{a}$ & $1,20 \mathrm{a}$ & $1,08 b c$ & $1,10 \mathrm{abc}$ & $1,06 \mathrm{c}$ & $1,17 \mathrm{ab}$ \\
\hline \multirow[t]{3}{*}{$\left(\mathrm{g} \mathrm{cm}^{-3}\right)$} & $10-15$ & $0,97 \mathrm{de}$ & $1,13 b$ & $1,11 b c$ & $1,24 \mathrm{a}$ & $1,07 \mathrm{bcd}$ & $0,96 \mathrm{e}$ & $1,15 \mathrm{ab}$ & $1,02 \mathrm{cde}$ \\
\hline & $20-25$ & $0,93 \mathrm{~d}$ & $1,04 b c$ & $1,02 \mathrm{bcd}$ & $1,05 b c$ & $1,08 \mathrm{ab}$ & $0,96 \mathrm{~cd}$ & $1,16 \mathrm{a}$ & $0,98 \mathrm{~cd}$ \\
\hline & $40-45$ & $0,98 \mathrm{~b}$ & $1,01 \mathrm{~b}$ & $1,03 \mathrm{~b}$ & $0,97 b$ & $1,16 \mathrm{a}$ & $0,99 b$ & $1,03 \mathrm{~b}$ & $1,05 \mathrm{~b}$ \\
\hline$\theta \mathrm{v}$ & $0-5$ & $0,35 \mathrm{ab}$ & $0,37 \mathrm{ab}$ & $0,37 \mathrm{ab}$ & $0,32 \mathrm{ab}$ & $0,29 b$ & $0,39 a$ & $0,37 a b$ & $0,43 \mathrm{a}$ \\
\hline \multirow[t]{3}{*}{$\left(\mathrm{cm}^{3} \mathrm{~cm}^{-3}\right)$} & $10-15$ & $0,34 \mathrm{a}$ & $0,32 \mathrm{a}$ & $0,37 \mathrm{a}$ & $0,33 a$ & $0,32 \mathrm{a}$ & $0,36 \mathrm{a}$ & $0,38 \mathrm{a}$ & $0,33 a$ \\
\hline & $20-25$ & $0,30 \mathrm{ab}$ & $0,30 \mathrm{ab}$ & $0,30 \mathrm{ab}$ & $0,28 \mathrm{ab}$ & $0,25 b$ & $0,34 \mathrm{ab}$ & $0,36 \mathrm{a}$ & $0,25 b$ \\
\hline & $40-45$ & $0,31 \mathrm{a}$ & $0,30 \mathrm{a}$ & $0,31 \mathrm{a}$ & $0,28 \mathrm{a}$ & $0,34 \mathrm{a}$ & $0,33 \mathrm{a}$ & $0,31 \mathrm{a}$ & $0,31 \mathrm{a}$ \\
\hline$\overline{\mathrm{RP}}$ & $0-5$ & $0,47 \mathrm{c}$ & $1,29 \mathrm{ab}$ & $1,65 \mathrm{a}$ & $1,47 \mathrm{a}$ & $0,19 \mathrm{c}$ & $1,10 \mathrm{ab}$ & $0,27 \mathrm{c}$ & $0,72 b c$ \\
\hline \multirow[t]{3}{*}{$(\mathrm{MPa})$} & $10-15$ & $0,73 b$ & $1,33 \mathrm{ab}$ & $1,67 \mathrm{a}$ & $1,67 \mathrm{a}$ & $0,73 b$ & $1,31 \mathrm{ab}$ & $0,79 b$ & $1,16 a b$ \\
\hline & $20-25$ & $0,98 \mathrm{c}$ & 1,39abc & $1,77 \mathrm{a}$ & $1,65 \mathrm{ab}$ & $1,12 b c$ & $1,41 \mathrm{abc}$ & $1,20 \mathrm{abc}$ & $1,53 \mathrm{abc}$ \\
\hline & $40-45$ & $1,03 \mathrm{~b}$ & $1,31 \mathrm{ab}$ & $1,72 \mathrm{a}$ & $1,69 \mathrm{a}$ & $1,19 \mathrm{ab}$ & $1,61 \mathrm{ab}$ & $1,18 \mathrm{ab}$ & $1,66 \mathrm{a}$ \\
\hline & $0-5$ & $0,68 \mathrm{a}$ & $0,58 \mathrm{~cd}$ & $0,54 d$ & $0,56 \mathrm{~cd}$ & $0,59 \mathrm{bc}$ & $0,58 \mathrm{bc}$ & $0,62 b$ & $0,56 \mathrm{~cd}$ \\
\hline \multirow{3}{*}{$\left(\mathrm{cm}^{3} \mathrm{~cm}^{-3}\right)$} & $10-15$ & $0,65 \mathrm{ab}$ & $0,58 \mathrm{de}$ & $0,59 \mathrm{cde}$ & $0,56 \mathrm{e}$ & $0,61 \mathrm{bcd}$ & $0,65 a$ & $0,58 \mathrm{de}$ & $0,62 \mathrm{abc}$ \\
\hline & $20-25$ & $0,68 \mathrm{a}$ & $0,63 b c$ & $0,64 b c$ & $0,62 \mathrm{c}$ & $0,61 \mathrm{c}$ & $0,66 a b$ & $0,57 \mathrm{~d}$ & $0,65 b c$ \\
\hline & $40-45$ & $0,65 \mathrm{ab}$ & $0,64 \mathrm{abc}$ & $0,61 \mathrm{~cd}$ & $0,66 \mathrm{a}$ & $0,58 \mathrm{~d}$ & $0,64 \mathrm{abc}$ & $0,62 \mathrm{bc}$ & $0,62 \mathrm{abc}$ \\
\hline \multirow{4}{*}{$\begin{array}{l}\text { MAC } \\
\left(\mathrm{cm}^{3} \mathrm{~cm}^{-3}\right)\end{array}$} & $0-5$ & $0,26 a$ & $0,07 b c$ & $0,05 \mathrm{c}$ & $0,06 \mathrm{bc}$ & $0,12 b$ & $0,06 \mathrm{bc}$ & $0,12 b$ & $0,08 b c$ \\
\hline & $10-15$ & $0,23 a$ & $0,12 b c$ & $0,15 b$ & $0,07 \mathrm{c}$ & $0,12 b c$ & $0,16 a b$ & $0,10 \mathrm{bc}$ & $0,16 a b$ \\
\hline & $20-25$ & $0,25 \mathrm{a}$ & $0,19 a b$ & $0,20 \mathrm{a}$ & $0,18 \mathrm{ab}$ & $0,12 b$ & $0,21 \mathrm{a}$ & $0,12 b$ & $0,18 \mathrm{ab}$ \\
\hline & $40-45$ & $0,22 \mathrm{a}$ & $0,21 \mathrm{a}$ & $0,19 a$ & $0,21 \mathrm{a}$ & $0,09 \mathrm{~b}$ & $0,16 \mathrm{ab}$ & $0,17 \mathrm{a}$ & $0,17 \mathrm{a}$ \\
\hline \multirow{4}{*}{$\begin{array}{l}\text { MIE } \\
\left(\mathrm{cm}^{3} \mathrm{~cm}^{-3}\right)\end{array}$} & $0-5$ & $0,15 \mathrm{c}$ & $0,18 \mathrm{ab}$ & $0,19 \mathrm{a}$ & $0,18 \mathrm{a}$ & $0,19 a$ & $0,18 \mathrm{abc}$ & $0,18 \mathrm{a}$ & $0,16 b c$ \\
\hline & $10-15$ & $0,12 \mathrm{c}$ & $0,15 b$ & $0,14 b c$ & $0,18 \mathrm{a}$ & $0,17 \mathrm{ab}$ & $0,16 a b$ & $0,16 a b$ & $0,16 a b$ \\
\hline & $20-25$ & $0,11 \mathrm{c}$ & $0,15 \mathrm{ab}$ & $0,13 b c$ & $0,18 \mathrm{a}$ & $0,16 a$ & $0,13 b c$ & $0,12 b c$ & $0,16 \mathrm{a}$ \\
\hline & $40-45$ & $0,13 \mathrm{c}$ & $0,17 \mathrm{a}$ & $0,14 \mathrm{bc}$ & $0,16 \mathrm{ab}$ & $0,15 \mathrm{abc}$ & $0,15 \mathrm{abc}$ & $0,14 \mathrm{abc}$ & $0,14 b c$ \\
\hline \multirow{4}{*}{$\begin{array}{l}\text { APD } \\
\left(\mathrm{cm}^{3} \mathrm{~cm}^{-3}\right)\end{array}$} & $0-5$ & $0,14 b$ & $0,16 a b$ & $0,16 a b$ & $0,17 \mathrm{a}$ & $0,17 \mathrm{a}$ & $0,16 a b$ & $0,16 \mathrm{a}$ & $0,14 a b$ \\
\hline & $10-15$ & $0,11 \mathrm{~d}$ & $0,13 \mathrm{bcd}$ & $0,12 \mathrm{~cd}$ & $0,16 \mathrm{a}$ & $0,15 \mathrm{ab}$ & $0,15 \mathrm{ab}$ & $0,14 \mathrm{abc}$ & $0,15 \mathrm{ab}$ \\
\hline & $20-25$ & $0,11 d$ & $0,14 b c$ & $0,12 \mathrm{~cd}$ & $0,16 a$ & $0,15 \mathrm{ab}$ & $0,12 \mathrm{~cd}$ & $0,11 \mathrm{~cd}$ & $0,15 \mathrm{ab}$ \\
\hline & $40-45$ & $0,13 \mathrm{~b}$ & $0,15 \mathrm{ab}$ & $0,13 \mathrm{ab}$ & $0,15 \mathrm{a}$ & $0,14 \mathrm{ab}$ & $0,14 a b$ & $0,13 \mathrm{ab}$ & $0,13 \mathrm{~b}$ \\
\hline
\end{tabular}

${ }^{(1)}$ Médias seguidas de mesma letra, na linha, não diferem entre si pelo teste de Tukey, a 5\% de probabilidade. ${ }^{(2)}$ Ds, densidade do solo; $\theta_{\mathrm{v}}$, umidade volumétrica do solo na amostragem; RP, resistência à penetração; PT, porosidade total; MAC: macroporosidade; MIE, microporosidade efetiva; APD, água prontamente disponível. 
subsuperficie, resultante da aração contínua do solo ao longo da condução do experimento.

No sistema lavoura contínua em plantio direto (L-D), observou-se acréscimo da Ds na camada de 0-5 cm, o que demonstra o efeito da compactação superficial, que pode ocorrer no plantio direto, devido ao não revolvimento do solo (Beutler et al., 2001). Porém, essa compactação não se propagou em profundidade, não tendo sido possível observar diferenças significativas em relação ao CER.

A umidade volumétrica foi influenciada pelos tratamentos e, em geral, observou-se maior conteúdo de água nas camadas de 0-5 e 10-15 cm e nos sistemas que apresentaram maior Ds (Tabela 2). Costa et al. (2003) observaram resultado semelhante ao comparar sistemas de preparo, em que o plantio direto apresentou maior umidade volumétrica na camada de $5 \mathrm{~cm}$. Os autores atribuíram esse resultado à maior infiltração e menor evaporação de água, decorrentes, respectivamente, da ausência de formação de selamento superficial e de cobertura do solo pela presença de resíduos.

A análise de correlação mostrou relação positiva e significativa entre Ds e $\theta_{\mathrm{v}}\left(\mathrm{r}=0,42^{*}\right)$. Apesar da presença de maior cobertura vegetal nos tratamentos em pastagem, a amplitude de variação de umidade entre os tratamentos foi pequena, mesmo ao comparar os outros sistemas com o LP-C, onde se espera maior evaporação da água devido à exposição pelo revolvimento do solo.

De acordo com Imhoff et al. (2000), a relação da resistência mecânica do solo à penetração com a umidade e densidade pode ser utilizada para fazer inferências sobre a condição estrutural e prever as relações entre a densidade crítica e o crescimento das raízes das plantas. Assim, foram estabelecidos modelos de RP de acordo com Ds e $\theta_{\mathrm{v}}$ (Busscher et al., 1997), que não foram significativos. $O$ resultado da análise de correlação entre essas variáveis não foi significativo $\left(\mathrm{r}=0,11^{\mathrm{ns}} \mathrm{e}-0,21^{\mathrm{ns}}\right.$ para as correlações de RP com Ds e $\theta_{\mathrm{v}}$, respectivamente). Este resultado provavelmente está relacionado ao fato de que os valores de $\theta_{\mathrm{v}}$ estavam próximos aos da capacidade de campo, e apresentaram pequena amplitude de variação (Tabela 2).

Os valores de RP foram influenciados pelos sistemas de manejo do solo e variaram em profundidade (Tabela 2). Nos sistemas em pastagem os valores de RP, assim como a Ds, foram superiores aos observados nos demais sistemas, sobretudo nas pastagens de Panicum maximum com quatro anos de idade. Entretanto, ao se adotar um valor crítico de RP para o crescimento do sistema radicular de culturas anuais e gramíneas entre 2,0 e 2,5 MPa (Taylor et al., 1966; Imhoff et al., 2000), observa-se que nenhum dos sistemas estudados apresentou resistência superior a este limite. Spera et al. (2004) avaliaram o impacto de sistemas agrícolas e de integração lavourapecuária sobre atributos físicos e observaram que a compactação resultante do pisoteio animal não interferiu negativamente nos atributos físicos, exceto pelo aumento da densidade do solo na fase pastagem.

Ao observar as curvas de $\theta_{\text {rel }}$ entre os diferentes sistemas, verifica-se, graficamente, tendência de aproximação das curvas com o aumento da profundidade (Figura 1), o que reforça a hipótese da similaridade do solo nas camadas mais profundas, a partir de $20-25 \mathrm{~cm}$, onde não haveria mais efeito dos sistemas de manejo e uso do solo. Verificou-se na camada superficial $(0-5 \mathrm{~cm})$ que todas as CRA dos sistemas de manejo foram significativamente diferentes da CRA do cerrado (Tabela 3). A partir da camada 10-15 cm, em geral, não houve diferença significativa entre os tratamentos sob ILP e a área sob cerrado (Figura 1), porém, observa-se, nesta camada, que o sistema PL-D foi significativamente diferente do cerrado, e não diferiu dos demais. Da mesma forma, nessa mesma profundidade, o tratamento sob pastagem contínua não diferiu de nenhum dos demais sistemas de cultivo. Tomando-se por base o ponto de tensão de $10 \mathrm{kPa}$, verificou-se aumento relativo no armazenamento de água no solo dos sistemas de ILP em relação ao cerrado, nas camadas superiores (Figura 1). As maiores diferenças foram observadas na camada inicial de $0-15 \mathrm{~cm}$, conforme o padrão dos resultados anteriormente mencionados. Nas profundidades de 20-25 e 40-45 cm, apenas o sistema LP-C apresentou comportamento distinto do cerrado, não diferindo dos demais sistemas, nem mesmo da pastagem contínua (Tabela 3 ).

Alguns trabalhos têm utilizado a curva de retenção de água do solo para comparar o efeito dos sistemas de manejo do solo (Carvalho et al., 1999; Oliveira et al., 2004), porém, em muitos casos, a comparação utilizando a curva como índice de qualidade física não leva a conclusões claras sobre o efeito do manejo.

$\mathrm{O}$ fato de englobar diferentes atributos em um mesmo modelo complexo, tais como os que expressam a retenção de água no solo, pode afetar a sensibilidade das curvas e inviabilizar sua utilização em comparações de sistemas de manejo. Todavia, devido a mudanças na forma da curva de retenção de água relativa com a degradação do solo, Dexter 
(2004) considera que a CRA tem potencial para ser utilizada como índice de qualidade física do solo em sistemas de uso e manejo que alterem mais drasticamente a distribuição de poros por tamanho, ou mesmo entre tipos de solos.
Na Figura 2, está representado o modelo gráfico comparativo da qualidade físico-hídrica do solo para a RP, Ds, MAC e MIE com os desvios relativos de cada parâmetro em relação ao cerrado. A Ds apresentou pequenas deformações relativas, mesmo na

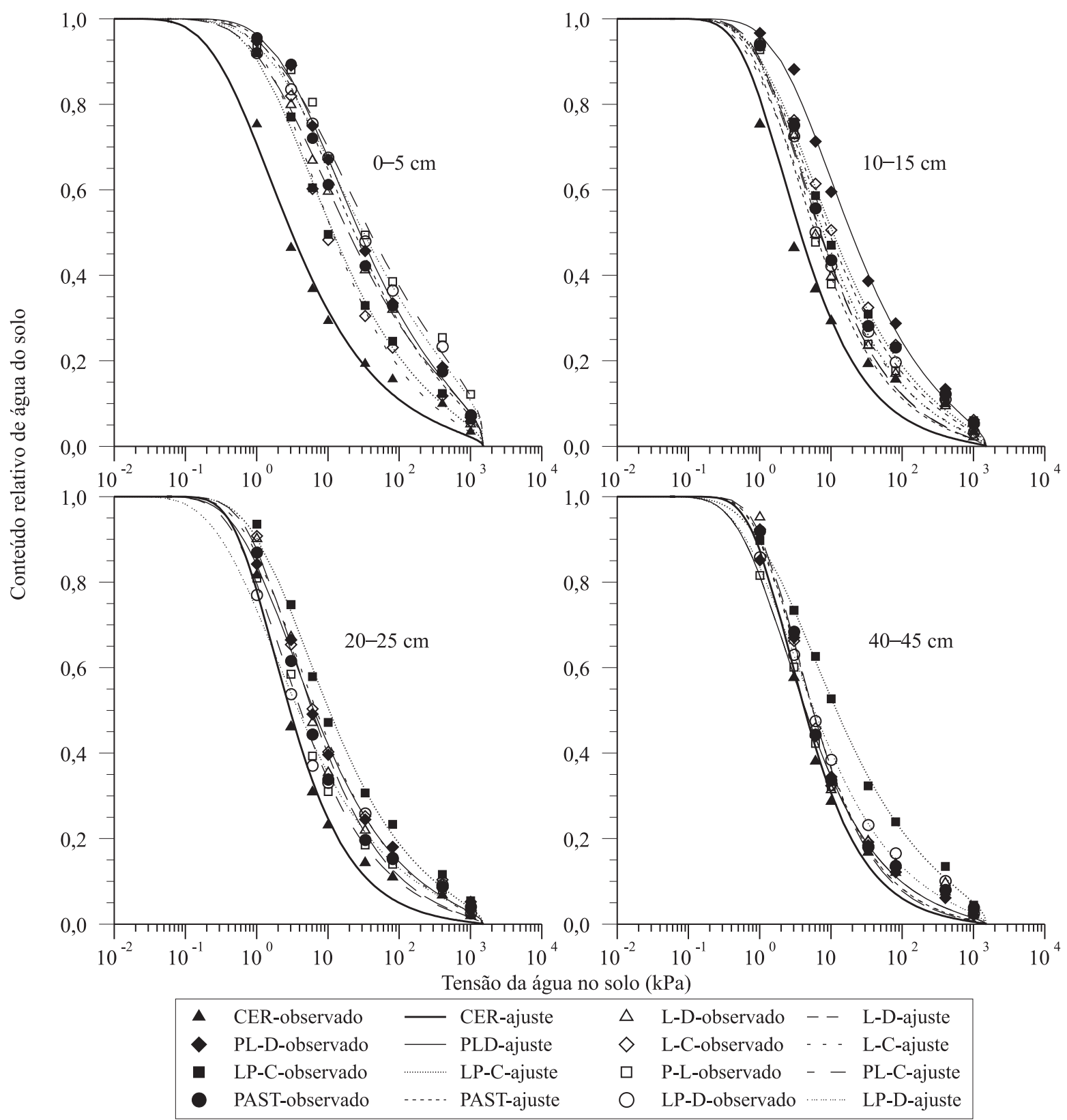

Figura 1. Curvas do conteúdo relativo versus tensão da água no solo em sistemas de integração lavoura-pecuária nas profundidades de 0-5, 10-15, 20-25 e 40-45 cm. CER, cerrado nativo; PAST, pastagem contínua de Brachiaria decumbens; PL-D, rotação pastagem-lavoura em plantio direto; PL-C, rotação pastagem-lavoura em plantio convencional; LP-D, rotação lavoura-pastagem em plantio direto; LP-C, rotação lavoura-pastagem em plantio convencional; L-D, lavoura contínua em plantio direto; L-C, lavoura contínua em plantio convencional. 
profundidade $0-5 \mathrm{~cm}$, porém o teste de médias (Tabela 2) mostra que a Ds foi significativamente superior à Ds observada no cerrado em todos os sistemas de manejo avaliados. Por sua vez, o eixo da RP foi o que apresentou maiores deformações até a profundidade de $40-45 \mathrm{~cm}$, indicando que, mesmo nessa profundidade, houve efeito dos sistemas de preparo do solo empregados. Na profundidade $0-5 \mathrm{~cm}$, os valores de RP foram superiores em todos os sistemas de manejo, exceto nos sistemas LP-C e L-C, que sofreram revolvimento e foram estatisticamente semelhantes aos observados no cerrado.

Entre os demais atributos físico-hídricos avaliados, somente a quantidade de poros insaturados calculada (PI) e a microporosidade (MIC) não foram influenciadas pelos tratamentos. Apesar de não promover incremento significativo na PI e na MIC, o plantio direto promove alterações no arranjo estrutural dos agregados que possivelmente lhe conferem maior microporosidade.

A MAC e a PT foram significativamente menores em todos os sistemas em comparação com o CER (Tabela 2). A redução foi de cerca de 80 e $20 \%$, ao comparar o cerrado com o sistema PL-C, na camada 0-5 cm, para MAC e PT, respectivamente. Na camada superficial $(0-5 \mathrm{~cm})$, todos os sistemas ocupados por pastagens (PAST e PL), incluindo a pastagem de $B$. brizantha na rotação (LP-D), apresentaram valores de MAC abaixo de $0,1 \mathrm{~cm}^{3} \mathrm{~cm}^{-3}$, que corresponde ao valor considerado crítico por Greenland (1981) como condição mínima de aeração para o desenvolvimento radicular.

O mesmo resultado foi observado no sistema lavoura contínua em plantio direto (L-D), conseqüência do aumento da densidade do solo em superfície. Assim como a RP, a MAC apresentou-se como um atributo sensível às alterações no solo decorrentes dos sistemas de manejo. As deformações nos polígonos ilustram a redução da MAC em relação ao cerrado em todas as profundidades avaliadas (Figura 2). Mesmo na camada mais profunda, 40-45 cm, a MAC foi sensível às alterações provocadas pelo sistema LP-C (Tabela 2). A drástica redução desse atributo nos solos cultivados decorre do aumento da compactação, que é evidenciada pelo aumento da densidade do solo (Corsini \& Ferraudo, 1999). Além disso, ela é fundamental na análise da distribuição de poros no solo por estar intimamente ligada à condutividade hidráulica e à porosidade ocupada com ar no potencial maior que $-6 \mathrm{kPa}$, sendo uma medida relacionada com a taxa de difusão de oxigênio no solo (Greenland, 1981). Deve-se considerar que a água não fica retida nas tensões correspondentes a MAC, devendo haver equilíbrio da relação entre macro e microporos.

Tabela 3. Valores de probabilidade do teste F, com 2 e 12 graus de liberdade, nas comparações das curvas do conteúdo relativo de água entre os sistemas de manejo, avaliados nas camadas de 0-5 e 10-15 cm (diagonal superior) e 20-25 e 40-45 cm (diagonal inferior) $)^{(1)}$.

\begin{tabular}{lcrrrrrrrrr}
\hline $\begin{array}{l}\text { Sistema } \\
\text { de } \\
\text { manejo }\end{array}$ & $\begin{array}{c}\text { Camada } \\
(\mathrm{cm})\end{array}$ & CER & PAST & PL-D & PL-C & LP-D & LP-C & $\begin{array}{c}\text { L-D } \\
\text { L-C }\end{array}$ & $\begin{array}{c}\text { Camada } \\
(\mathrm{cm})\end{array}$ \\
\hline CER & - & - & 0,0000 & 0,0000 & 0,0000 & 0,0000 & 0,0001 & 0,0000 & 0,0003 \\
& - & - & 0,0443 & 0,0000 & 0,9328 & 0,1959 & 0,0068 & 0,3283 & 0,0016 & $10-15$ \\
PAST & $20-25$ & 0,9104 & - & 30,5013 & 1,0497 & 5,8702 & 13,5810 & 4,5064 & 0,0877 & $0-5$ \\
& $40-45$ & 15,2054 & - & 0,0318 & 19,8660 & 53,9796 & 26,9538 & 15,0141 & 21,3614 & $10-15$ \\
PL-D & $20-25$ & 0,0030 & 5,8896 & - & 2,8145 & 8,2808 & 0,0002 & 0,0357 & 0,0039 & $0-5$ \\
& $40-45$ & 16,9208 & 55,8367 & - & 0,0035 & 0,0049 & 0,0230 & 0,0005 & 0,0310 & $10-15$ \\
PL-C & $20-25$ & 1,9425 & 17,8518 & 0,1564 & - & 24,9327 & 0,0096 & 0,0151 & 0,0011 & $0-5$ \\
& $40-45$ & 17,9771 & 3,7658 & 11,1624 & - & 69,2827 & 0,1577 & 96,7117 & 0,6565 & $10-15$ \\
LP-D & $20-25$ & 1,1781 & 3,8130 & 0,2570 & 25,4502 & - & 0,0002 & 0,0790 & 0,0027 & $0-5$ \\
& $40-45$ & 0,0112 & 7,6555 & 9,4935 & 1,0508 & - & 13,4545 & 66,1572 & 2,1653 & $10-15$ \\
LP-C & $20-25$ & 0,0001 & 10,5928 & 0,0548 & 0,0325 & 0,0016 & - & 0,0371 & 49,4926 & $0-5$ \\
& $40-45$ & 0,0003 & 20,4555 & 0,0001 & 0,0022 & 0,0003 & - & 1,7443 & 54,4083 & $10-15$ \\
L-D & $20-25$ & 0,0319 & 35,4686 & 25,3555 & 1,6662 & 0,5762 & 0,1674 & - & 0,2259 & $0-5$ \\
& $40-45$ & 20,7715 & 97,0114 & 52,1340 & 6,4683 & 10,8399 & 0,0171 & - & 0,1737 & $10-15$ \\
L-C & $20-25$ & 0,0057 & 6,0240 & 59,7883 & 0,2323 & 0,2129 & 1,1143 & 16,5634 & - & - \\
& $40-45$ & 15,8132 & 98,9567 & 60,7344 & 3,5578 & 6,5377 & 0,0008 & 93,7916 & - & - \\
\hline
\end{tabular}

(1)CER: cerrado nativo; PAST, pastagem contínua de Brachiaria decumbens; PL-D, rotação pastagem-lavoura em plantio direto; PL-C, rotação pastagem-lavoura em plantio convencional; LP-D, rotação lavoura-pastagem em plantio direto; LP-C, rotação lavoura-pastagem em plantio convencional; L-D, lavoura contínua em plantio direto; L-C, lavoura contínua em plantio convencional. 

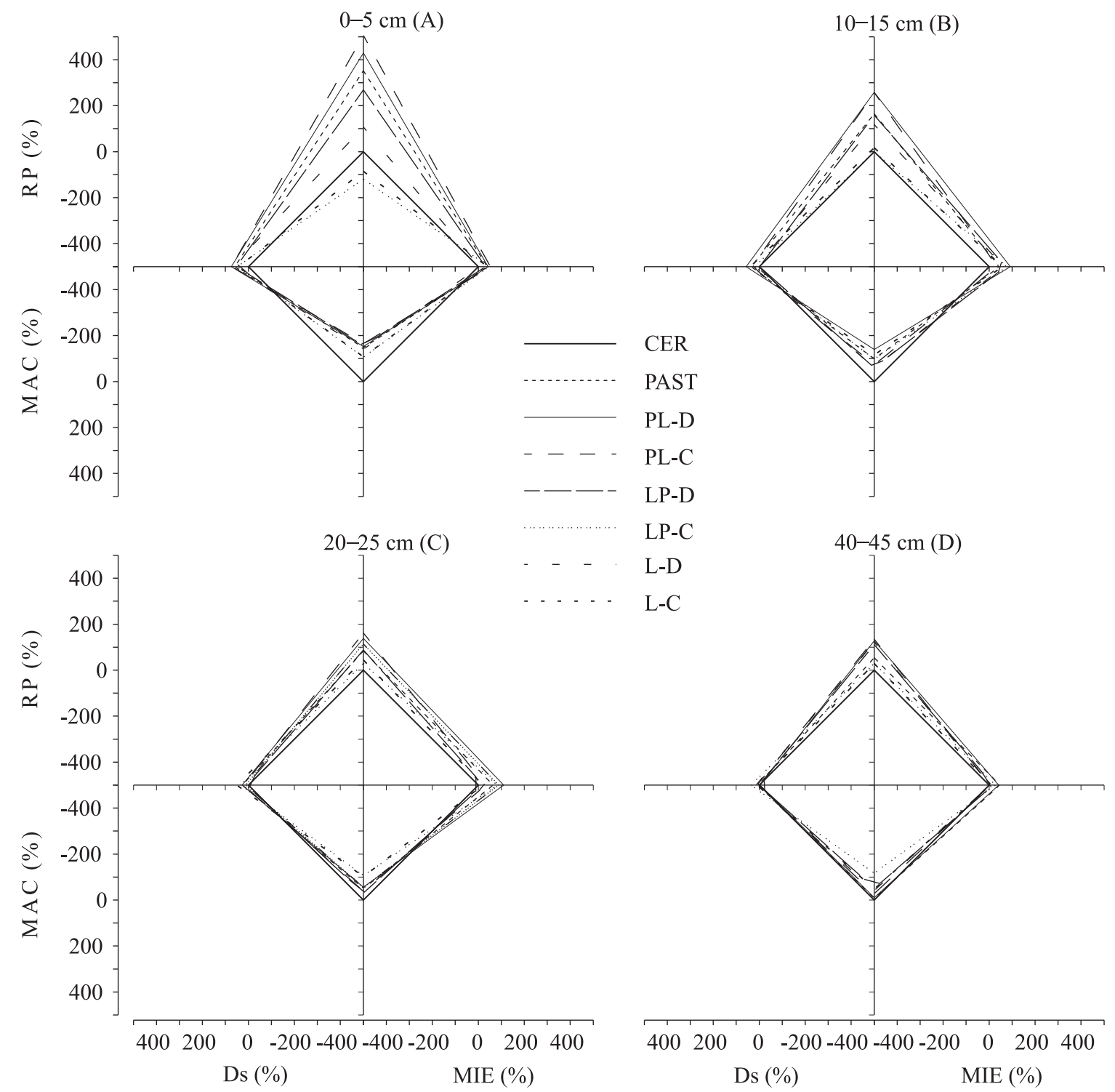

Figura 2. Modelo gráfico comparativo da qualidade físico-hídrica do solo em sistema de integração lavourapecuária, relativo ao cerrado (CER) nas camadas 0-5 cm (A); 10-15 cm (B); 20-25 cm (C); e 40-45 cm (D), considerando os valores relativos dos parâmetros resistência à penetração (RP), macroporosidade (MAC), densidade do solo (Ds) e microporosidade efetiva (MIE).

Os valores de MIE foram significativamente superiores aos observados no cerrado em todos os sistemas de manejo, o que promoveu aumento na água prontamente disponível (APD) (Tabela 2). A MIE e a APD expressam a capacidade do solo em armazenar e disponibilizar água para as plantas e são atributos importantes na Região do Cerrado. Nesta região, a redução da disponibilidade de água no solo pode prejudicar ou até mesmo limitar o desenvolvimento de algumas forrageiras (Muller et al., 2002) e pode se tornar um fator limitante no estabelecimento de pastagens de inverno. Nos sistemas de ILP, um aumento na capacidade de armazenamento de água do solo é fundamental para o sucesso das culturas de sucessão implantadas que tem por objetivo fornecer forragem na entressafra. 


\section{Conclusões}

1. Todos os sistemas de integração lavoura-pecuária provocam impacto na qualidade físico-hídrica do solo, na profundidade de $0-5 \mathrm{~cm}$, em relação à condição original observada no cerrado.

2. A curva de retenção de água do solo pode ser utilizada na comparação de sistemas de manejo e também como indicadora da qualidade físico-hídrica do solo.

3. O gráfico comparativo da qualidade físico-hídrica do solo permite visualizar as modificações impostas pelos sistemas de manejo.

\section{Agradecimentos}

Aos assistentes de pesquisa Wantuir Caixeta e Nelson Camargos Moreira, da Embrapa Cerrados; ao Institut de Recherche pour le Developpement, França, pela concessão de bolsa de doutorado ao primeiro autor.

\section{Referências}

ASSOULINE, S.; TESSIER, D.; BRUAND, A. A conceptual model of the soil water retention curve. Water Resources Research, v.34, p.223-231, 1998.

BALBINO, L.C.; BRUAND, A.; BROSSARD, M.; GUIMARÃES, M.F. Comportement de la phase argileuse lors de la dessication dans des Ferralsols microagrégés du Brésil: rôle de la microstructure et de la matiére organique. Comptes Rendus de l'Académie des Sciences, v.332, p.673-680, 2001.

BALBINO, L.C.; BRUAND, A.; COUSIN, I.; BROSSARD, M.; QUÉTIN, P.; GRIMALDI, M. Change in the hydraulic properties of a Brazilian clay Ferralsol on clearing for pasture. Geoderma, v.120, p.297-307, 2004.

BEUTLER, A.N.; SILVA, M.L.N.; CURI, N.; FERREIRA, M.M.; CRUZ, J.C.; PEREIRA FILHO, I.A. Resistência à penetração e permeabilidade de Latossolo Vermelho distrófico típico sob sistemas de manejo na região dos cerrados. Revista Brasileira de Ciência do Solo, v.25, p.167-177, 2001.

BUSSCHER, W.J.; BAUER, P.J.; CAMP, C.R.; SOJKA, R.E. Correction of cone index for soil water content differences in a coastal plain soil. Soil \& Tillage Research, v.43, p.205-217, 1997.

CARVALHO, E.J.M.; FIGUEIREDO, M.S.; COSTA, L.M. Comportamento físico-hídrico de um Podzólico Vermelho-Amarelo câmbico fase terraço sob diferentes sistemas de manejo. Pesquisa Agropecuária Brasileira, v.34, p.257-265, 1999.

CHAPUIS LARDY, L.; BROSSARD M.; LOPES ASSAD, M.L.; LAURENT, J.Y. Carbon and phosphorus stocks of clayey Ferralsols in Cerrado native and agroecosystems, Brazil. Agriculture, Ecosystems and Environment, v.92, p.147-158, 2002.
COSTA, E.A.; GOEDERT, W.J.; SOUSA, D.M.G. de. Qualidade de solo submetido a sistemas de cultivo com preparo convencional e plantio direto. Pesquisa Agropecuária Brasileira, v.41, p.11851191, 2006.

COSTA, F.S.; AlbUQUERQUE, J.A.; BAYER, C.; FONTOURA, S.M.V.; WOBETO, C. Propriedades físicas de um Latossolo Bruno afetadas pelos sistemas plantio direto e preparo convencional. Revista Brasileira de Ciência do Solo, v.27, p.527-535, 2003.

CORSINI, P.C.; FERRAUDO, A.S. Efeitos de sistemas de cultivo na densidade e macroporosidade do solo e no desenvolvimento radicular do milho em Latossolo Roxo. Pesquisa Agropecuária Brasileira, v.34, p.289-298, 1999.

DEXTER, A.R. Soil physical quality: part III. Unsaturated hydraulic conductivity and general conclusions about S-theory. Geoderma, v.120, p.227-239, 2004.

EMBRAPA. Centro Nacional de Pesquisas de Solos (Rio de Janeiro). Manual de métodos de análises de solo. 2.ed. Rio de Janeiro, 1997. 212p.

EMBRAPA. Centro Nacional de Pesquisa de Solos (Rio de Janeiro). Sistema Brasileiro de Classificação de solos. 2.ed. Rio de Janeiro, 2006. 306p.

GARCÍA-PRÉCHAC, F.; ERNST, O.; SIRI-PRIETO, G.; TERRA, J.A. Integrating no-till into crop-pasture rotations in Uruguay. Soil \& Tillage Research, v.77, p.1-13, 2004.

GREENLAND, D.J. Soil management and soil degradation. Journal of Soil Science, v.31, p.301-322, 1981.

IMHOFF, S.; SILVA, A.P.; TORMENA, C.A. Applications of the resistance curve in the control of the physical quality of soils under grass. Pesquisa Agropecuária Brasileira, v.35, p.1493-1500, 2000.

LAL, R. Physical management of the soils of the tropics: priorities for the $21^{\text {st }}$ century. Soil Science, v.165, p.191-207, 2000.

LEÃO, T.P.; SILVA, A.P.; MACEDO, M.C.M.; IMHOFF, S.; EUCLIDES, V.P.B. Least limiting water range: a potential indicator of changes in near-surface soil physical quality after the conversion of Brazilian Savanna into pasture. Soil \& Tillage Research, v.88, p.279-285, 2006.

MARCHÃO, R.L. Integração lavoura-pecuária num latossolo do cerrado: impacto na física, matéria orgânica e macrofauna. 2007. 153p. Tese (Doutorado) - Universidade Federal de Goiás, Goiânia.

MILLENNIUM ECOSYSTEM ASSESSMENT - MEA. Ecosystems and human well-being: general synthesis report. Washington: Island Press, 2005. Disponível em: <http:// www.millenniumassessment.org/en/Synthesis.aspx $>$ Acessado em 7/5/2007.

MULLER, M.S.; FANCELLI, A.L.; DOURADO-NETO, D.; GARCÍA, A.G.; OVEJERO, R.F.L. Produtividade do Panicum maximum cv. Mombaça irrigado, sob pastejo rotacionado. Scientia Agricola, v.59, p. 427-433, 2002.

OLIVEIRA, G.C.; DIAS JUNIOR, M.S.; RESCK, D.V.S.; CURI,

N. Caracterização química e físico-hídrica de um Latossolo Vermelho 
após vinte anos de manejo e cultivo do solo. Revista Brasileira de Ciência do Solo, v.28, p.327-336, 2004.

REYNOLDS, W.D.; BOWMAN, B.T.; DRURY, C.F.; TAN, C.S.; LU, X. Indicators of good soil physical quality: density and storage parameters. Geoderma, v.110, p.131-146, 2002.

SANO, E.E.; BARCELLOS, A.O.; BEZERRA, H.S. Assessing the spatial distribution of cultivated pastures in the Brazilian savanna. Pasturas Tropicales, v.22, p.2-15, 2000.

SILVA, E.M. da; AZEVEDO, J.A. Influência do período de centrifugação na curva de retenção de água em solos de Cerrado. Pesquisa Agropecuária Brasileira, v.37, p.1487-1494, 2002.

SILVA, E.M. da; LIMA, J.E.F.W.; AZEVEDO, J.A. de; RODRIGUES, L.N. Valores de tensão na determinação da curva de retenção de água de solos do Cerrado. Pesquisa Agropecuária Brasileira, v.41, p.323-330, 2006.
SILVA, J.E.; RESCK, D.V.S. Respostas fisiológicas da soja ao déficit hídrico em dois solos de Cerrado. Pesquisa Agropecuária Brasileira, v.16, p.669-675, 1981.

SPERA, S.T.; SANTOS, H.P.; FONTANELI, R.S.; TOMM, G.O. Efeitos de sistemas de produção de grãos envolvendo pastagens sob plantio direto nos atributos físicos de solo e na produtividade. Revista Brasileira de Ciência do Solo, v.28, p.533-542, 2004.

TAYLOR, H.M.; ROBERSON, G.M.; PARKER, J.J. Soil strength-root penetration relations to coarse textured materials. Soil Science, v.102, p.18-22, 1966.

TORMENA, C.A.; SILVA, A.P.; LIBARDI, P.L. Soil physical quality of a Brazilian Oxisol under two tillage systems using the least limiting water range approach. Soil \& Tillage Research, v.52, p.223-232, 1999.

$\overline{\text { Recebido em } 28 \text { de fevereiro de } 2007 \text { e aprovado em } 11 \text { de maio de } 2007}$ 